\title{
Group B streptococcal $\beta$-hemolysin/cytolysin activates neutrophil signaling pathways in brain endothelium and contributes to development of meningitis
}

\author{
Kelly S. Doran, George Y. Liu, and Victor Nizet \\ Department of Pediatrics, Division of Infectious Diseases, University of California, San Diego, School of Medicine, \\ La Jolla, California, USA
}

\begin{abstract}
Meningitis occurs when blood-borne pathogens cross the blood-brain barrier (BBB) in a complex interplay between endothelial cells and microbial gene products. We sought to understand the initial response of the BBB to the human meningeal pathogen group B Streptococcus (GBS) and the organism's major virulence factors, the exopolysaccharide capsule and the $\beta$-hemolysin/cytolysin toxin $(\beta-h / c)$. Using oligonucleotide microarrays, we found that GBS infection of human brain microvascular endothelial cells (HBMEC) induced a highly specific and coordinate set of genes including IL-8, Gro $\alpha$, Gro $\beta$, IL-6, GM-CSF, myeloid cell leukemia sequence-1 (Mcl-1), and ICAM-1, which act to orchestrate neutrophil recruitment, activation, and enhanced survival. Most strikingly, infection with a GBS strain lacking $\beta-\mathrm{h} / \mathrm{c}$ resulted in a marked reduction in expression of genes involved in the immune response, while the unencapsulated strain generally induced similar or greater expression levels for the same subset of genes. Cell-free bacterial supernatants containing $\beta-h / c$ activity induced IL-8 release, identifying this toxin as a principal provocative factor for BBB activation. These findings were further substantiated in vitro and in vivo. Neutrophil migration across polar HBMEC monolayers was stimulated by GBS and its $\beta-\mathrm{h} / \mathrm{c}$ through a process involving IL-8 and ICAM-1. In a murine model of hematogenous meningitis, mice infected with $\beta-\mathrm{h} / \mathrm{c}$ mutants exhibited lower mortality and decreased brain bacterial counts compared with mice infected with the corresponding WT GBS strains.
\end{abstract}

J. Clin. Invest. 112:736-744 (2003). doi:10.1172/JCI200317335.

\section{Introduction}

Bacterial meningitis is the most common serious infection of the CNS. Blood-borne pathogens must interact with cerebral endothelial cells and cross the bloodbrain barrier (BBB); subsequent bacterial replication within the CNS may provoke an overwhelming host inflammatory response. While in vitro and in vivo models have been informative about the pathogenesis of bacterial meningitis and CNS factors that contribute to inflammation and brain injury, little is known about the specific contribution of the BBB endothelium to the initial threat of an invading pathogen.

The Gram-positive bacterium group B Streptococcus (GBS) is the leading cause of meningitis in newborn infants. Morbidity is high despite antibiotic therapy; $25-50 \%$ of surviving infants suffer neurological sequelae of varying severity, including cerebral palsy,

Received for publication November 7, 2002, and accepted in revised form June 17, 2003.

Address correspondence to: Kelly S. Doran, Division of Infectious Diseases, University of California, San Diego, School of Medicine, 9500 Gilman Drive, Mail Code 0672, La Jolla, California 92093-0672, USA. Phone: (858) 534-7477;

Fax: (858) 534-7411; E-mail: kdoran@ucsd.edu.

Conflict of interest: The authors have declared that no conflict of interest exists.

Nonstandard abbreviations used: blood-brain barrier (BBB); group B Streptococcus (GBS); human brain microvascular endothelial cell(s) (HBMEC); $\beta$-hemolysin/cytolysin ( $\beta$-h/c); ToddHewitt broth (THB); myeloid cell leukemia sequence-1 (Mcl-1). mental retardation, deafness, or seizures (1). Several studies in animals and humans have shown that high-level bacteremia is required for the development of meningitis (2); however, for GBS the precise mechanism (or mechanisms) whereby the bacterium leaves the bloodstream and gains access to the CNS remains unclear. It is likely that GBS tropism for human brain microvascular endothelial cells (HBMEC) is the primary step in the pathogenesis of meningitis, whereupon a combination of bacterial transcytosis, endothelial cell injury, and inflammatory mechanisms combine to disrupt the BBB (3).

Two proven GBS virulence factors predicted to impact the pathogenesis of meningitis are the surface polysaccharide capsule and $\beta$-hemolysin/cytolysin ( $\beta$-h/c). In all GBS serotypes, the capsule contains sialic acid and acts as a primary bloodstream survival factor, promoting the establishment of bacteremia by inhibiting activation of the alternative pathway of complement and thereby interfering with neutrophil opsonophagocytic killing mechanisms. The GBS $\beta-\mathrm{h} / \mathrm{c}$ is a pore-forming membrane-associated toxin that promotes injury of a broad range of eukaryotic cell types $(4,5)$, including HBMEC (3), and contributes to disease progression and virulence in animal models $(6,7)$. Encapsulation and/or cytolytic exotoxins are shared features of all major bacterial agents of human meningitis, including Streptococcus pneumoniae, Neisseria meningitidis, Haemophilus influenzae, Escherichia coli $\mathrm{K} 1$, and Listeria monocytogenes (8). 
In this study we examine the acute response of the $\mathrm{BBB}$ to a bacterial pathogen using oligonucleotide microarray, real-time RT-PCR, and protein analysis. We identify differentially expressed genes from a human brain endothelial cell line infected with WT GBS and show that the BBB plays an active role in initiating a very specific innate immune response promoting neutrophil recruitment. Experiments with isogenic GBS mutants lacking capsule or the $\beta-\mathrm{h} / \mathrm{c}$ toxin demonstrate that these virulence factors impact the level of gene induction in strikingly different fashions. Our studies suggest that BBB endothelium responds specifically to the bacterial $\beta-h / c$ toxin with functional gene expression to promote the characteristic neutrophilic inflammatory response of acute bacterial meningitis.

\section{Methods}

Bacterial strains and supernatants. The WT clinical GBS isolates used in these studies were $\mathrm{COH} 1$, a highly encapsulated type III strain; A909, a type Ia strain; and NCTC10/84 (1169-NT1), a highly hemolytic type V strain. The capsule-deficient mutant HY106 (9) is a single-gene allelic exchange derivative of $\mathrm{COH} 1$, as are the $\beta$-h/c-deficient mutant $\left(\beta-\mathrm{h} / \mathrm{c}^{-}\right)$strains (COH1:cylE $\Delta$ cat, A909:cylE $\Delta$ cat, and NCTC:cylE $\Delta$ cat) of each respective WT strain (10). Because the target gene has been deleted from the bacterium, these mutants are stable, unlike mutations created with transposons or integrative plasmids that are capable of excision and reversion to the WT phenotype. All strains grew equally well under the conditions used in these experiments. For preparing supernatants containing $\beta-\mathrm{h} / \mathrm{c}$ activity, the highly hemolytic WT strain NCTC10/84 and its $\beta$-h/c-deficient derivative NCTC:cylE $\Delta$ cat were used. GBS-stabilized supernatants were prepared essentially as described previously (4), with a few modifications. Briefly, GBS was grown in Todd-Hewitt broth (THB) supplemented with $0.2 \%$ glucose to mid-log phase $\left(\sim 10^{8} \mathrm{CFU} / \mathrm{ml}\right.$; $\left.\mathrm{OD}_{600}=0.4\right)$, at which point the cells were harvested and resuspended in PBS containing $2 \%$ starch and $0.2 \%$ glucose to extract the $\beta$-h/c activity from the bacterial surface. After incubation for 1 hour at $37^{\circ} \mathrm{C}$, the supernatant was collected, filtered, added to cold $100 \%$ methanol at a vol/vol ratio of $1: 1$, and incubated 1 hour at $-20^{\circ} \mathrm{C}$. The supernatant was then centrifuged and the pellet was resuspended in PBS, yielding an approximate hemolytic activity of $50 \mathrm{U} / \mu \mathrm{l}$ as determined by hemoglobin release (4); $25 \mathrm{U}$ was used to induce IL-8 expression.

Human BBB model. The human brain microvascular endothelial cell line HBMEC, which has been immortalized by transfection with the SV40 large T antigen (11), was obtained from Kwang Sik Kim (Johns Hopkins University, Baltimore, Maryland, USA). HBMEC maintain the morphologic and functional characteristics of primary brain endothelial cells $(12,13)$. The cells were maintained in RPMI 1640 medium (Life Tech- nologies Inc., Grand Island, New York, USA) supplemented with 10\% FBS, 10\% NuSerum (Becton, Dickinson and Co., Bedford, Massachusetts, USA), MEM nonessential amino acids, and penicillin-streptomycin and were incubated at $37^{\circ} \mathrm{C}$ in $5 \% \mathrm{CO}_{2}$. For microarray experiments, real-time RT-PCR and ELISA, cells were used at passage 8 ; for other experiments including invasion, cell-free IL-8 induction, and transendothelial migration, cells were used at passage 8-14.

Tissue culture infections. For the microarray experiments, within 24 hours of establishing confluence ( $\sim 10^{5}$ cells per well), HBMEC monolayers were washed twice with PBS, and then RPMI 1640 plus $10 \%$ FBS was added. GBS strains COH1, HY106, and COH1:cylE $\Delta-$ cat were grown in THB to mid-log phase $\left(\sim 10^{8}\right.$ $\left.\mathrm{CFU} / \mathrm{ml} ; \mathrm{OD}_{600}=0.4\right)$, washed in PBS, resuspended in RPMI plus $10 \%$ FBS, and used to infect HBMEC monolayers at an MOI of ten bacteria per cell (10:1). Plates were centrifuged at $800 \mathrm{~g}$ for 5 minutes to place bacteria on the monolayer surface, then incubated at $37^{\circ} \mathrm{C}$ in $5 \% \mathrm{CO}_{2}$ for 1,2 , 4 , or 8 hours. All three GBS strains were capable of invading HBMEC, and the differences in invasion levels between the strains were not significant (data not shown). Although the presence of GBS capsule typically appears to attenuate the ability of GBS to invade HBMEC (3), the capsule mutant used in these studies did not appear to differ significantly in invasion efficiency compared with the WT strain under the conditions used for these experiments. After infection for the indicated times, the supernatant was removed from the monolayer, centrifuged to remove bacteria, and stored at $-20^{\circ} \mathrm{C}$ for further analysis. HBMEC monolayers were also collected and stored at $-70^{\circ} \mathrm{C}$ for RNA isolation.

GeneChip bybridization and analysis. Total RNA was isolated from cell monolayers at various timepoints using the RNeasy miniprep kit (Qiagen Inc., Valencia, California, USA) according to the manufacturer's protocol and digested with DNase I to remove contaminating genomic DNA. One microgram total RNA was used for double-stranded cDNA synthesis using the Superscript Choice System (Invitrogen Corp., San Diego, California, USA) according to the manufacturer's directions. Synthesis of biotin-labeled cRNA was carried out by in vitro transcription using the MEGAscript T7 in vitro transcription kit (Ambion Inc., Austin, Texas, USA). The biotin-labeled cRNA was fragmented and hybridized to the U95 human GeneChip array (Affymetrix Inc., Santa Clara, California, USA) following the manufacturer's instructions. GeneChip arrays were scanned with the GeneArray Scanner (HewlettPackard, Santa Clara, California, USA) controlled by GeneChip 3.1 software (Affymetrix Inc.). Data was analyzed using a statistical algorithm developed for highdensity oligonucleotide arrays (14).

Real-time kinetic RT-PCR and immunoassays. The level of expression of specific transcripts was determined using the ABI Prism 7700 and Sequence Detection System software (Applied Biosystems, Foster City, Cali- 
Table 1

HBMEC transcription profile after infection with GBS

\begin{tabular}{llc}
\multicolumn{2}{c}{ Chemokines and cytokines } & \\
M28130 & IL-8 & Fold change $^{A}$ \\
M36820 & GRO- $\beta$ chemokine & 61.6 \\
X54489 & GRO- $\alpha$ chemokine & 42 \\
X04430 & IL-6 & 22.6 \\
M13207 & GM-CSF & 13.6 \\
\end{tabular}

Cell-cell interaction

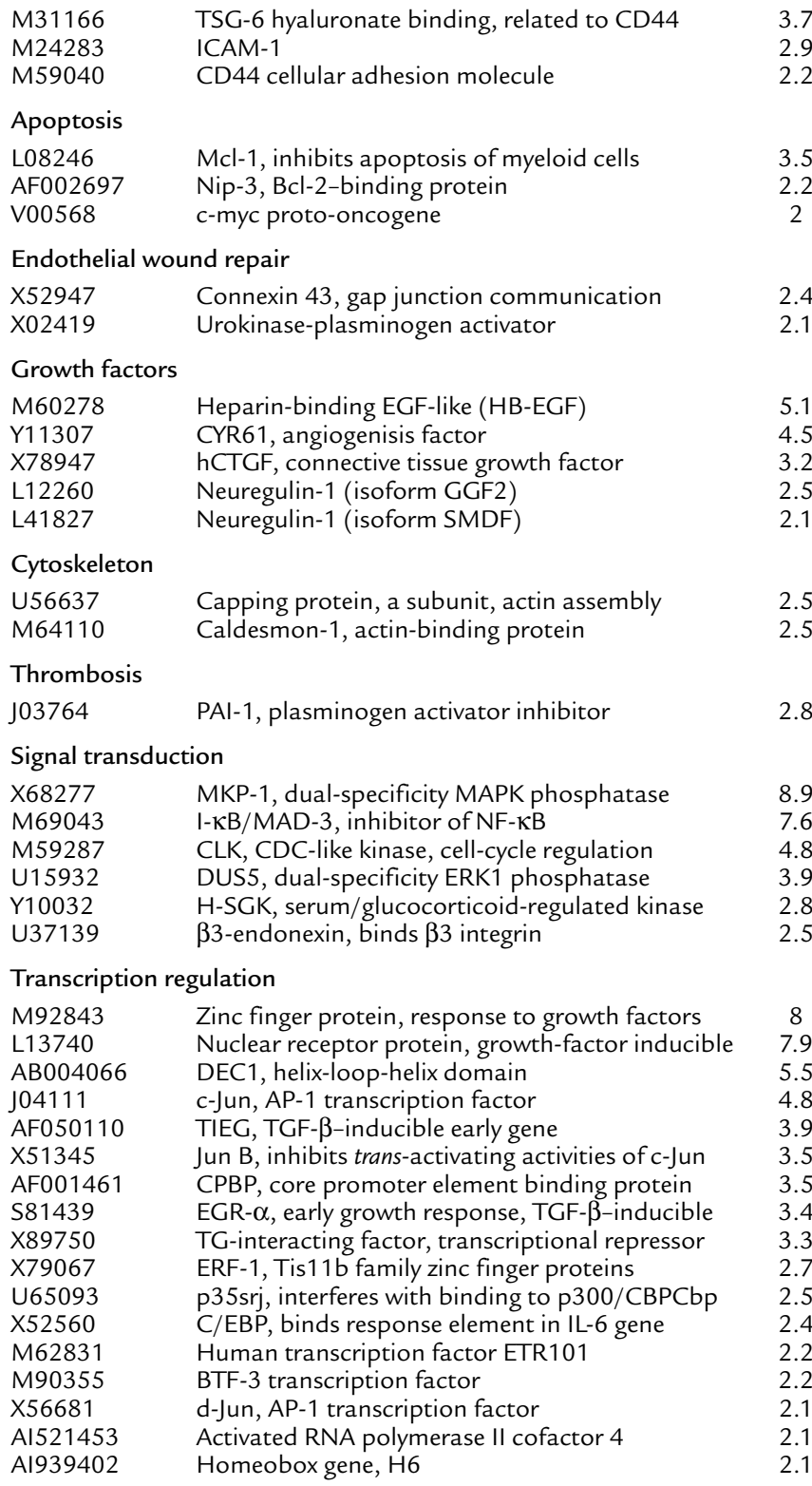

${ }^{A}$ Ratio of gene expression of infected vs. unifected cells. Values reported are increased above background twofold or more at 4 hours.

fornia, USA). IL-8 protein secretion was measured by ELISA as described previously $(15,16)$; IL-1 $\beta$, IL-6, Gro $\alpha$, GM-CSF, and TNF- $\alpha$ protein secretion were analyzed using commercially available ELISA kits (R\&D Systems Inc., Minneapolis, Minnesota, USA).

Neutrophil transendothelial migration assay. Human neutrophils were purified from a healthy donor using a
Histopaque gradient (Sigma-Aldrich, St. Louis, Missouri, USA) according to the manufacturer's directions. Following centrifugation, the granulocytes were harvested and contaminating erythrocytes were lysed with $155 \mathrm{mM} \mathrm{NH}_{4} \mathrm{Cl}, 10 \mathrm{mM} \mathrm{KHCO}_{3}$, and $0.1 \mathrm{mM}$ EDTA. Neutrophil purity was greater than $95 \%$. Polar HBMEC monolayers were established on collagen-coated Transwell plates (Transwell-COL; CorningCostar Corp., Acton, Massachusetts, USA) as described previously (3), except that the membrane used in these experiments had a pore size of $3 \mu \mathrm{m}$. Monolayers were incubated with the WT or the $\beta$-h/c mutant strain $(\mathrm{MOI}=10: 1)$ for 4 hours as described above before the assay was initiated. Alternatively, $0.5 \mathrm{ml}$ of cell-free conditioned supernatant obtained from HBMEC monolayers previously infected with WT or the $\beta$-h/c mutant was added to the lower chamber. Supernatant from cells that were not exposed to bacteria served as a control. Neutrophils $\left(1 \times 10^{5}\right)$ were added to the upper chamber and plates were incubated for 2 hours at $37^{\circ} \mathrm{C}$ in $5 \% \mathrm{CO}_{2}$, after which migrated cells in the lower chamber were counted on a hemocytometer. For inhibition studies, $10 \mu \mathrm{g} / \mathrm{ml}$ human IL-8-specific rabbit antibody (Perbio Science AB, Helsingborg, Sweden), $10 \mu \mathrm{g} / \mathrm{ml}$ human ICAM-1specific mouse antibody (R\&D Systems Inc.), and $10 \mu \mathrm{g} / \mathrm{ml}$ of mouse IgG1 isotype control antibody (R\&D Systems Inc.) were added to the upper and lower chambers 1 hour prior to the start of the assay.

Murine model of hematogenous GBS meningitis. Outbred 6-week-old male CD-1 mice were obtained from Charles River Laboratories (Wilmington, Massachusetts, USA). Mice were injected via the tail vein with $10^{8}$ CFU of two WT GBS strains of differing $\beta$-h/c activity, A909 (four hemolytic units) and NCTC10/84 (32 to 64 hemolytic units), respectively (4), and the corresponding isogenic $\beta$-h/c mutant for each strain (10). Twenty-four hours after injection, blood from the tail vein was obtained and plated on THB agar plates to ensure bacteremia and success of injection. Seventy-two hours after infection, mice were euthanized, their brains were aseptically removed, and the hemispheres were separated. Half of the brain was homogenized in sterile PBS and half was fixed in formalin and embedded in paraffin for sectioning and histopathologic analysis. Bacterial counts in the brain homogenates and blood were determined by plating serial tenfold dilutions on THB agar plates. Brain bacterial counts were corrected for blood contamination 
using the blood concentration and a conservative estimate of the mouse cerebral blood volume $(2.5 \mathrm{ml}$ per $100 \mathrm{~g}$ tissue, as extrapolated from the rat) (17). The significance of differences between treatment groups was determined using the unpaired Student $t$ test. $P<0.05$ was considered to be significant.

\section{Results}

Expression profile of the $B B B$ induced by GBS. Understanding the response of the $\mathrm{BBB}$ endothelium to the threat of an invading bacterium should provide insight into the first line of CNS defense and the virulence attributes of meningeal pathogens. We used microarray analysis as a preliminary survey to examine the transcriptional responses of HBMEC 1, 2, 4, and 8 hours after infection with WT GBS. Previous analysis had shown that infection for more than 8 hours resulted in cellular cytotoxicity largely attributable to the activity of the GBS $\beta-h / c$. By 4 hours after infection, 80 HBMEC genes exhibited a greater than twofold change in transcript abundance. Table 1 categorizes the most notable of these HBMEC genes and their 4-hour induction values. The most highly induced HBMEC genes were IL-8, Gro $\alpha$, and Gro $\beta$, which belong to the CXC subfamily of chemokines. These molecules share a high affinity for receptors found on neutrophils and are known to act as strong neutrophil chemoattractants. Other HBMEC genes induced by GBS infection possess well-known roles in neutrophil inflammatory responses. GM-CSF stimulates the proliferation, differentiation, and migration of granulocytes and macrophages. ICAM-1 plays an essential role as an endothelial receptor to bind and activate circulating neutrophils at the site of infection. Myeloid cell leukemia sequence-1 (Mcl-1) is an antiapoptotic factor related to Bcl-2 that serves to promote neutrophil survival (18). Another proinflammatory cytokine induced was IL-6, which mediates a variety of immune responses and is elevated in the cerebrospinal fluid of patients with meningitis. Other upregulated gene products, such as I- $\kappa \mathrm{B}$, are induced by NF- $\mathrm{KB}$ signaling, which is involved in regulating these chemokines and cytokines. However, genes for many other proinflammatory markers produced by leukocytes, tissue macrophages, or epithelial cells in response to bacterial pathogens (e.g., TNF- $\alpha$, IL- $1 \alpha$, and inducible nitric oxide synthase) were not induced in HBMEC by GBS during the acute interaction. The overall gene expression profile of GBS-infected HBMEC suggests a relatively specific and rapid activation of the host innate defense system for neutrophil signaling.

Differential expression profiles induced by capsule and $\beta$ - $h / c-$ deficient $G B S$ strains. Capsular polysaccharide and the $\beta-\mathrm{h} / \mathrm{c}$ toxin are two major GBS virulence factors that might play a role in the observed pattern of HBMEC gene activation. The contribution of these factors was assessed using the same microarray analysis described in Methods, except that HBMEC were infected with isogenic capsule-negative and $\beta$-h/c-negative mutants of the WT GBS strain COH1. Table 2 shows the effects of these two mutations specifically on some of the genes most highly induced by the WT strain, most of which participate in the immune response. Absence of the capsule had a variable effect on the level of HBMEC gene induction; most of the mutant genes showed induction levels similar to the WT genes. In contrast, absence of the $\beta-\mathrm{h} / \mathrm{c}$ toxin resulted in a consistent decrease in HBMEC gene induction levels compared with the WT strain. For several of the induced genes implicated in promoting a neutrophilic inflammatory response, a pattern emerged in which the $\beta$-h/c-deficient mutant induced less transcript and the capsule-deficient mutant induced more transcript than the WT GBS strain. The microarray data show that mRNA levels for various housekeeping genes like $\beta$-actin, 18S rRNA, and GAPDH were similar for samples infected with WT or mutant GBS strains (data not shown). Additionally, there were ten genes that were induced twofold or more after infection with the $\beta$-h/c-deficient mutant compared with the WT strain. These included hypothetical protein AA522530, transcription factor U49436, transmembrane protein AB015631, IFN-related developmental regulator Y10313, GTT1 protein AL041780, catenin X87838, and inhibitor of DNA binding X77956).

Analysis of specific genes and gene products by real-time kinetic RT-PCR and ELISA. To continue our analysis and confirm our microarray expression data by an independent method, we assayed by real-time kinetic RT-PCR the relative abundance of five mRNA's that had different expression profiles in the HBMEC infected with WT, $\beta$-h/c-deficient mutant, and capsule-deficient mutant GBS strains. Figure 1 shows the mRNA levels of IL-8, Gro $\alpha$, Gro $\beta$, IL- 6 , and GM-CSF 4 hours after infection; all four genes were expressed at significantly higher levels in WT-infected cells than in either the

\section{Table 2}

Effect of GBS mutations on immune response genes

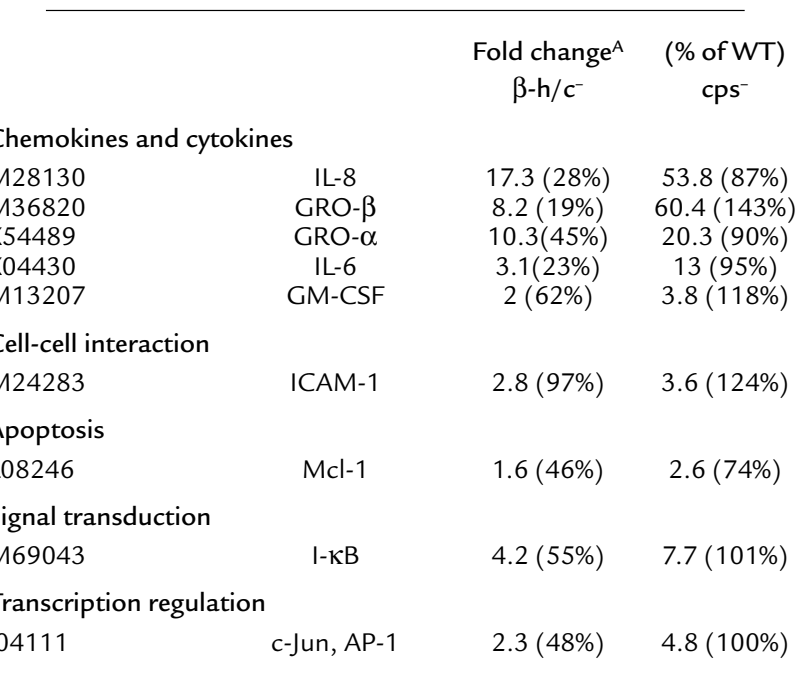

ARatio of gene expression of infected vs. uninfected cells at 4 hours. 


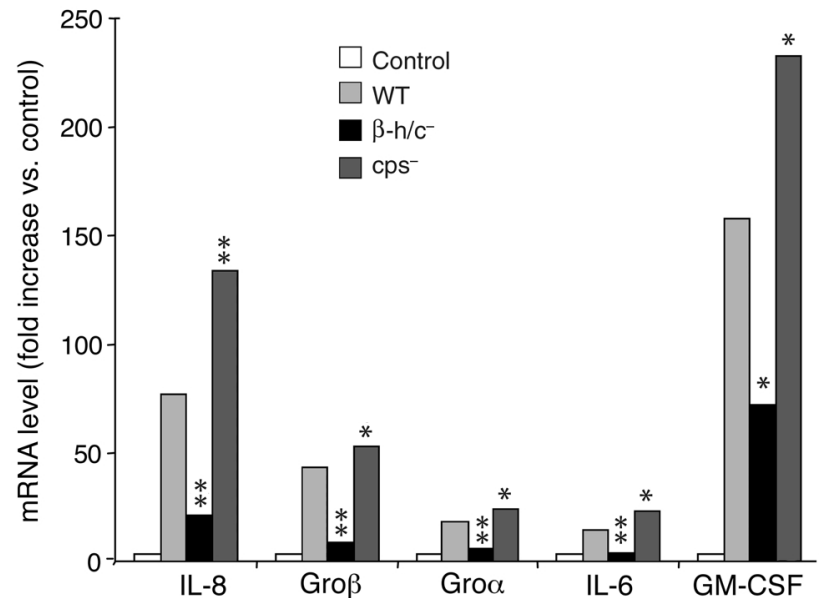

Figure 1

Effect of GBS mutations on transcript levels. We used real-time RT-PCR to verify the differential expression for five transcripts (IL-8, Gro $\beta$, Gro $\alpha$, IL- 6 , and GM-CSF) after infection with WT, $\beta$-h/c-deficient $\left(\beta-h / c^{-}\right)$, or capsule-deficient $\left(\mathrm{cps}^{-}\right)$GBS. The levels of IL-8, IL-6, Gro $\alpha$, and GM-CSF message were normalized to the 18S rRNA standard and Gro $\beta$ to GAPDH. Representative data from duplicate experiments are shown. The mean of triplicate wells resulted in SDs of less than \pm 1.5 in all cases. ${ }^{*} P<0.05$ compared with WT. **P $P 0.001$ compared with WT.

$\beta$-h/c mutant-infected or uninfected cells. The capsule mutant-infected cells expressed higher mRNA levels than those with WT infection. In general, the relative abundance of the different transcripts correlated with the fold increases observed by the microarray analysis. No increases in IL- $1 \beta$ or TNF- $\alpha$ transcription were detected by RT-PCR analysis (data not shown).

We further analyzed the protein expression responses for several of the chemokine and cytokine genes that were differentially induced in HBMEC by WT and mutant GBS. Figure 2 shows the increase in secreted IL-8, IL-6, Gro $\alpha$, and GM-CSF in supernatants 4 hours after GBS infection. Induction of chemokine and cytokine secretion was markedly reduced when cells were infected with the GBS $\beta-h / c$ mutant, while secretion was somewhat increased when cells were infected with the GBS capsule mutant, especially for IL-8 and GM-CSF. In all cases the 4-hour timepoint proved most reliable due to evidence of cellular cytotoxicity at 8 hours after infection, although cytokine secretion did increase for all the proteins measured at the 8-hour timepoint (data not shown). ELISA for IL- $1 \beta$ and TNF- $\alpha$ showed no increase in HBMEC secretion of these cytokines in response to GBS (data not shown).

Direct effect of GBS $\beta-h / c$ on stimulation of IL-8 release from HBMEC. Compared with the WT strain, the GBS mutant lacking the $\beta-\mathrm{h} / \mathrm{c}$ toxin exhibited a significantly decreased ability to induce mRNA transcript and protein secretion of key neutrophil signaling factors. We sought to determine whether the $\beta-\mathrm{h} / \mathrm{c}$ toxin could act independently to induce HBMEC gene expression, specifically that of IL-8. Cell-free supernatants from GBS were prepared in PBS plus $2 \%$ starch to extract stabilized $\beta$-h/c activity from the bacterial surface as described in Methods. HBMEC monolayers were incubated with WT bacteria and cellfree supernatants from either WT or $\beta$-h/c mutant strains. Figure 3 shows that supernatant containing $\beta$-h/c induced IL-8 release at levels comparable to infection with WT bacteria. These results indicate that the GBS $\beta-\mathrm{h} / \mathrm{c}$ exerts a stimulatory effect independent of bacterial contact with the HBMEC surface. In control experiments, supernatant from a $\beta-h / c$ mutant induced only background levels of IL-8 similar to the uninfected control, demonstrating that other secreted GBS products had negligible stimulatory effects. It should be noted that the hemolytic titer of the supernatant used was greater than that of the WT COH1 bacteria used (see ref. 4). Therefore, while the supernatant containing $\beta$-h/c activity was capable of inducing IL-8, the whole bacterium may provide a stronger stimulus due to additional bacterial factors that potentiate $\beta-\mathrm{h} / \mathrm{c}$ activity in its normal context on the GBS surface.

GBS-induced gene products promote transendothelial migration of human neutrophils. The HBMEC genes and gene products that were most highly induced in response to GBS infection in our microarray and protein studies are involved in the recruitment and activation of neutrophils. To examine the functional
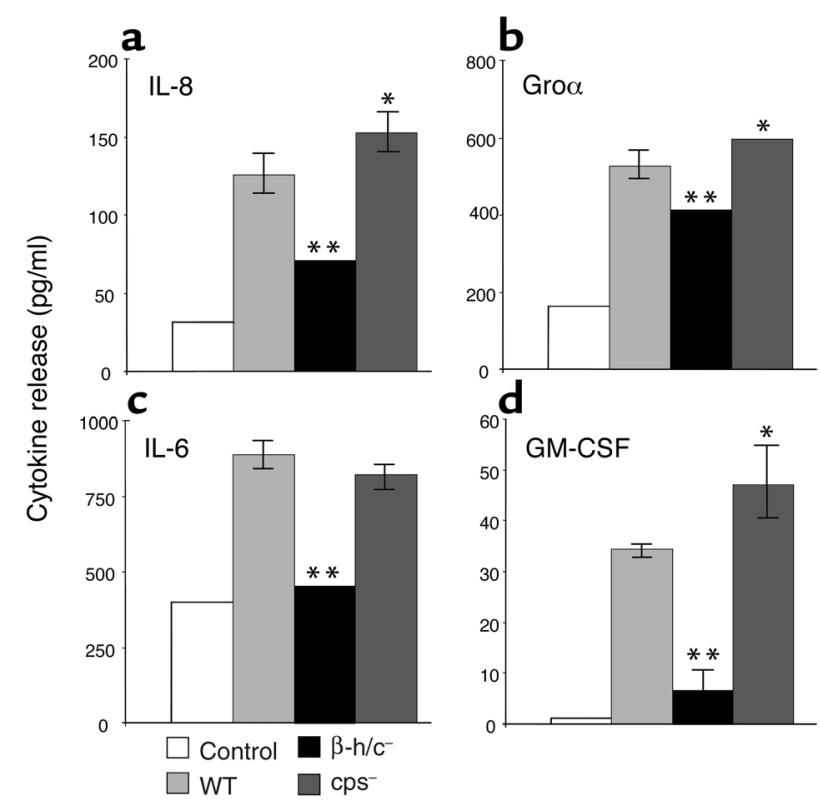

\section{Figure 2}

Changes in HBMEC chemokine and cytokine secretion after infection with WT, $\beta$-h/c-deficient, or capsule-deficient GBS. Cell culture supernatants were assayed after 4 hours of incubation with GBS. Assays were performed for IL-8 (a), Gro $\alpha(\mathbf{b})$, IL-6 (c), and GM-CSF (d). Representative data from duplicate experiments are shown. Error bars represent the $95 \%$ confidence interval of the mean of six wells. Error bars that are not visible represent an SD of less than \pm 1.5 . ${ }^{*} P<0.05$ compared with WT. ${ }^{* * P}<0.001$ compared with WT. 


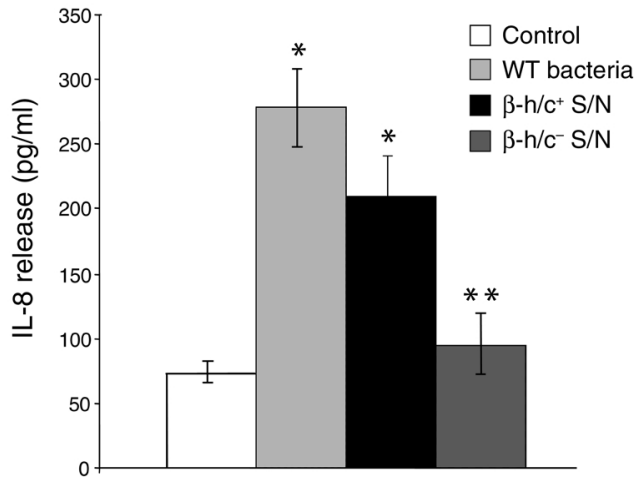

Figure 3

Stimulation of IL-8 release by HBMEC with supernatant $(\mathrm{S} / \mathrm{N})$ containing GBS $\beta$-h/c activity. Cell culture supernatants were assayed 4 hours after incubation with WT GBS, stabilized supernatant $(\sim 25 \cup)$ from $W T\left(\beta-h / c^{+}\right)$GBS, and stabilized supernatant from $\beta-h / c$ mutant $\left(\beta-h / c^{-}\right)$GBS. Representative data from duplicate experiments are shown. Error bars represent the $95 \%$ confidence interval of the mean of four wells. ${ }^{*} P<0.001$ compared with control. ${ }^{*} P<0.0001$ compared with WT.

chemoattractant activity of the GBS-induced factors, an in vitro model of neutrophil transendothelial migration was developed. Polar monolayers of HBMEC were established on Transwell inserts and infected with either WT or $\beta$-h/c mutant GBS. Human neutrophils were subsequently added to the upper chamber of the Transwell. WT GBS infection significantly increased neutrophil migration across an HBMEC monolayer compared with uninfected cells or cells infected with the $\beta$-h/c mutant (Figure $4 a$ ). Similar results were observed when HBMEC-conditioned supernatants (supernatant from HBMEC previously infected with WT or the $\beta-\mathrm{h} / \mathrm{c}$ mutant strain) were added to the lower chamber of the Transwell (Figure $4 \mathrm{~b})$. To assess the specific role of the neutrophil chemoattractant IL-8 and the neutrophil adhesin ICAM-1 in migration, we added specific antibodies against each host factor 1 hour before the addition of the neutrophils. Both antibodies significantly reduced neutrophil migration, while an isotype control antibody did not, indicating that GBS induced HBMEC expression of functional IL-8 and ICAM-1. Live bacteria are likely to produce different levels of neutrophil activation and/or neutrophil injury than are produced by cell-free HBMEC-conditioned supernatants, thus we are cautious not to draw conclusions from the slight increase in migration seen with supernatants and the greater effect of inhibitory antibodies observed with live bacteria. Statistically significant stimulatory effects of $\beta-\mathrm{h} / \mathrm{c}$ and inhibitory effects of anti-IL-8 and anti-ICAM- 1 antibodies were seen in both experimental systems.

Role of the $\beta$ - $h / c$ toxin in a murine model of GBS meningitis. Our microarray analysis and neutrophil transmigration studies suggested a prominent role for the GBS $\beta-\mathrm{h} / \mathrm{c}$ toxin in the pathogenesis of BBB penetration and meningitis. To test this hypothesis in vivo, we used the murine model of hematogenous streptococcal meningitis (19). Groups of mice $(n=10-13)$ were infected with one of two different WT GBS strains (A909 and NCTC10/84) that possess varying amounts of $\beta$-h/c activity (A909 has less activity than NCTC10/84), together with the corresponding isogenic $\beta$-h/c-deficient mutants. Seventy-two hours after infection, the mouse brains were harvested, homogenized, serially diluted, and plated on THB agar for quantitative bacterial counts (CFU). As shown in Figure 5a, each GBS WT strain penetrated the $\mathrm{BBB}$ and established meningitis more frequently than the corresponding isogenic $\beta$ - $\mathrm{h} / \mathrm{c}$-deficient mutants did (50\% vs. $30 \%$ for A909, $92 \%$ vs. $58 \%$ for NCTC10/84). Among WT strains, mortality and mean bacterial colony counts in the brain increased as the level of $\beta-\mathrm{h} / \mathrm{c}$ activity increased (compare $20 \%$ mortality and $1.7 \times 10^{2} \mathrm{CFU} / \mathrm{g}$ for A909 with $62 \%$ mortality and $1 \times 10^{6} \mathrm{CFU} / \mathrm{g}$ for the hyperhemolytic strain NCTC10/84). The mean bacterial colony counts isolated from the brains of mice infected with $\beta$-h/c-deficient mutants compared with the parent

\section{Figure 4}

Transmigration of neutrophils in response to bacterial infection (a) and supernatants of GBS-infected HBMEC (b). In duplicate experiments, neutrophils migrated in response to GBS-induced gene products by the WT or $\beta$-h/c-deficient strain with or without $10 \mu \mathrm{g} / \mathrm{ml}$ anti-IL-8, anti-ICAM-1, or IgG1 isotype control antibody. Migration index is the ratio of cells that migrated during an infection or in response to a supernatant $(\mathrm{S} / \mathrm{N})$ in comparison with migration across an uninfected monolayer. Error bars represent the $95 \%$ confidence interval of the mean of three wells. ${ }^{*} P<0.05$ compared with WT. ${ }^{* *} P<0.001$ compared with WT or the $\beta$-h/c mutant.
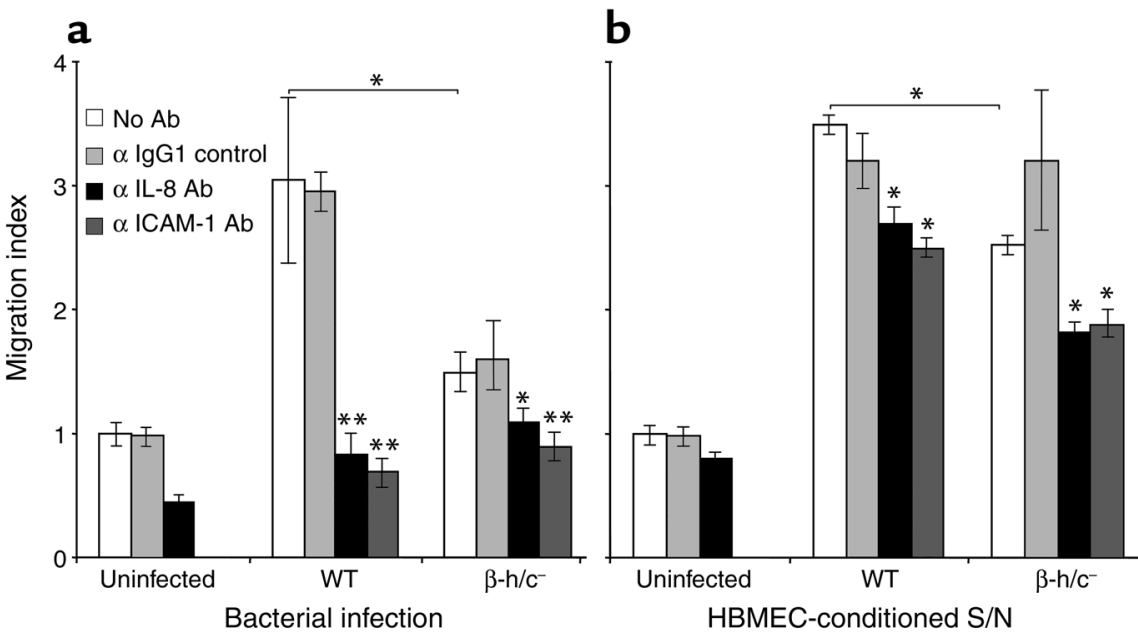

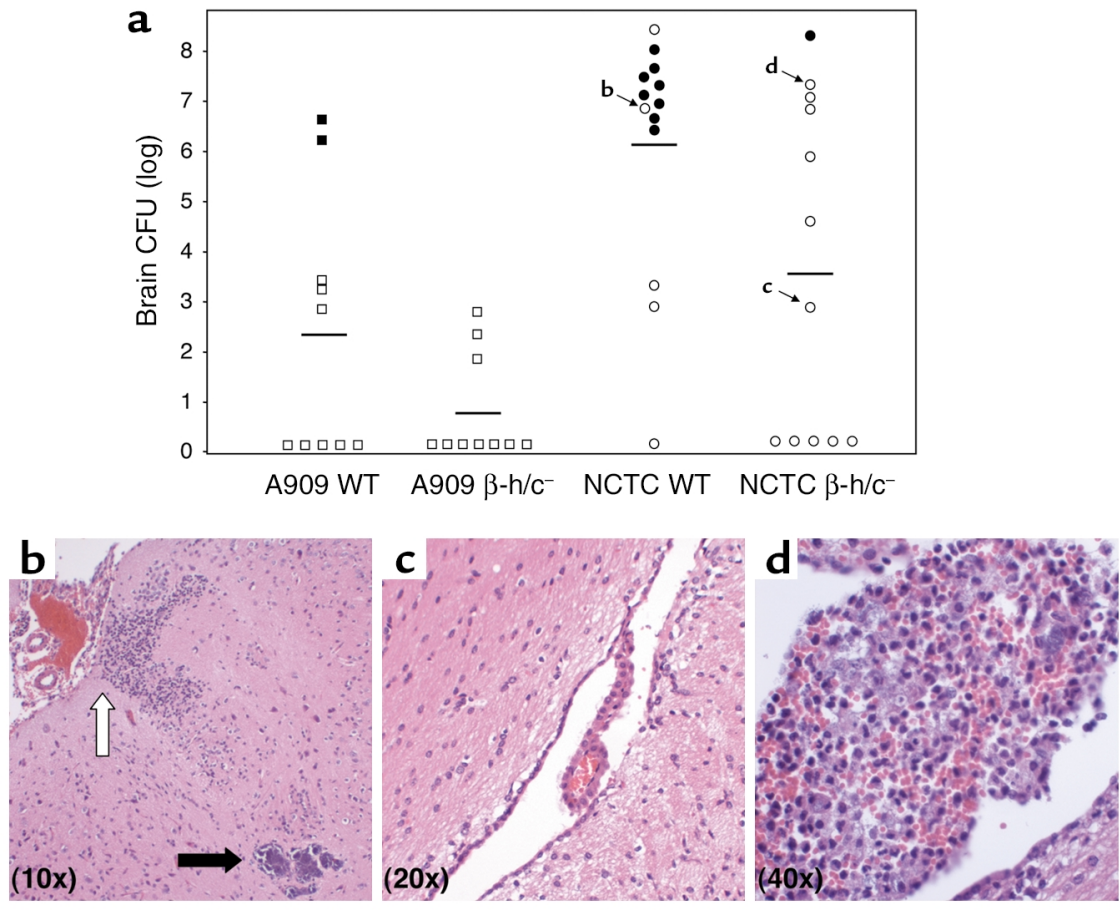

\section{Figure 5}

Murine model of GBS meningitis. (a) Groups of 10-13 mice were inoculated intravenously with GBS strains of increasing $\beta$-h/c activity (A909 and NCTC10/84, respectively) and the corresponding $\beta-\mathrm{h} / \mathrm{c}^{-}$ mutants. Seventy-two hours after infection, brains were harvested and bacterial counts (CFU/g brain) were determined. Solid shapes represent animals that died prior to the 72-hour timepoint. Letters with arrows indicate the mouse whose histopathology appears in panels b-d. (b-d) Histopathology of H\&E-stained brain tissues of individual mice (identified by arrows) that developed meningitis. (b) Mouse infected with WT GBS with high CNS bacterial counts showing meningeal thrombosis, thickening, and cellular infiltration (open arrow) as well as bacterial microabscess formation (solid arrow). (c) Mouse infected with $\beta-\mathrm{h} / \mathrm{c}^{-}$ mutant with low CNS bacterial counts and normal brain histopathology. (d) Neutrophilic and monocytic infiltrates typical of mice with high-grade CNS invasion with either WT or mutant GBS strains. strain were 34-fold lower for A909 $(P=0.05)$ and 250fold lower for NCTC10/84 $(P=0.02)$. These data indicate that $\beta$-h/c contributes to the risk and magnitude of GBS penetration of the BBB.

Representative histopathology of brains from experimentally infected mice are shown in Figure 5, b-d. For all groups, the degree of histopathologic abnormality correlated with the magnitude of the bacterial counts isolated from the brain. Figure 5 b shows a section from a mouse infected with WT GBS that developed meningitis and ventriculitis with brain bacterial counts near the group mean. Meningeal thickening, thrombosis of meningeal vessels, leukocytic infiltration, and areas of bacterial microabscess formation in the brain parenchyma were observed. In contrast, a section from a mouse infected with an isogenic $\beta-\mathrm{h} / \mathrm{c}-$ deficient mutant with low brain bacterial counts near the group mean showed normal appearance of the brain parenchyma and meningeal lining (Figure 5c). However, expression of $\beta$-h/c was not an absolute requirement for development of CNS pleocytosis, since in a few cases mice did develop high-grade CNS invasion with a GBS $\beta$-h/c mutant and showed evidence of meningeal infiltration with neutrophils and monocytes qualitatively similar to that observed in the WT infection (Figure 5d).

\section{Discussion}

The expression profile of GBS-infected BBB endothelium was probed initially by DNA microarray analysis, which served to guide subsequent detailed studies using real-time PCR and immunoassays. Only 80 of the 12,000 genes represented on the array exhibited an increase in gene transcription levels of twofold or greater 4 hours after infection. Only five genes, each unrelated to known inflammatory response pathways, exhibited a twofold or greater decrease in gene transcription levels (transcription factor AL080102; transcription factor U49436; lamin B2, M94362; laminin receptor M14199; annexin A2, M62895). A disproportionate amount of upregulated versus downregulated genes appears to be a common feature of host cellular responses to microbial pathogens, in particular those genes implicated in immune defense (20-23).

A variety of factors can induce endothelial activation, including cytokines, endotoxin, shear stress, hypoxia, infections, and lipid products $(24)$. The activation of the BBB endothelium by GBS resulted in the upregulation primarily of proinflammatory cytokines functioning to recruit inflammatory cells to the site of infection and injury (Table 1). The most highly induced genes - IL-8, Gro $\alpha$, and Gro $\beta$ belong to the CXC chemokine family, which acts mainly on cells of neutrophil lineage (24). IL-8 is the most potent chemotactic factor for neutrophils because it has a high affinity for both of the chemokine receptors (CXCR1 and CXCR2) expressed on neutrophils, as opposed to the Gro proteins, which possess affinity only for CXCR2 (25). In addition, IL-8 stimulates neutrophil respiratory burst, degranulation, and adherence to endothelial cells. Other GBS-induced HBMEC genes related specifically to CNS neutrophil recruitment were ICAM-1, which when upregulated leads to the enhanced adhesion of neutrophils to the brain endothelium (26), and GM-CSF, which increases neutrophil migration across brain endothelium (27). The programmed cell 
death of neutrophils can be delayed by GM-CSF (28-30), a process that was recently shown to be mediated through the specific induction of the antiapoptotic factor Mcl-1 (31), whose gene product was also elevated in our experiments. Our data suggest that GBS promotes leukocyte infiltration into the cerebrospinal fluid (a long recognized clinical and diagnostic hallmark of acute bacterial meningitis) through the upregulation of chemokines, which significantly increased neutrophil migration across an HBMEC monolayer.

Notably absent from the activation profile were several proinflammatory cytokines, including IL-1 and TNF- $\alpha$, which are involved in the pathogenesis of bacterial meningitis; both mediate inflammation by stimulating the production of other cytokines including IL-8, IL-6, and GM-CSF, the upregulation of the cell adhesion molecule ICAM-1, and BBB permeability (8). RT-PCR and ELISA studies confirmed the absence of TNF- $\alpha$ and IL- $1 \beta$ message and secreted protein in the acute response of GBS-stimulated HBMEC. The ability of BBB endothelium to produce targeted recruitment and activation of neutrophils in response to the initial bacterial encounter may be an important adaptation, since a wider or unchecked pattern of inflammatory activation could be deleterious to critical CNS structures sensitive to intracranial pressure changes. A proportionate localized neutrophil phagocytic response may be effective in the vast majority of $\mathrm{BBB}$ encounters with bacteria circulating in the cerebral microvasculature. Our findings that HBMEC do not produce TNF- $\alpha$ or IL-1 in response to an acute GBS infection suggests that cells other than the brain endothelium, including astrocytes, microglial cells, and recruited leukocytes, may be the main source of these cytokines during disease progression. In fact, TNF- $\alpha$ induction may be more significant to the latter stages of meningitis, because it contributes to apoptosis of hippocampal neurons (32) and further breakdown of BBB integrity (33).

Recognition of bacteria and neutrophil recruitment may be a key barrier function of HBMEC, acting as the very first line of CNS defense. But why does the innate protection mechanism fail in some cases, and what are the bacterial factors that contribute to this failure? To address this question we assessed the role of two major GBS virulence factors on the HBMEC response profile. Compared to WT GBS infection, we found that infection of HBMEC with a $\beta$-h/c-negative mutant resulted in significantly less gene induction and secretion of key immune mediator proteins. Neutrophil migration across an HBMEC monolayer was reduced in the presence of the $\beta-\mathrm{h} / \mathrm{c}$ mutant compared with the WT strain. Additionally, $\beta$ - $h / c$ mutants were less virulent than WT GBS strains in a murine model of meningitis. Thus $\beta-\mathrm{h} / \mathrm{c}$ appears to be a key mediator in provoking an acute inflammatory response in the brain endothelium and an important contributor to disease progression. Whether this action is elicited by direct interaction of the toxin with endothelial signal transduction systems or activation is a secondary result of cellular injury that is mediated by the toxin remains to be elucidated. Recent studies investigating other bacterial/host interactions have shown that various cytokine responses can be stimulated by toxins from E. coli (STEC) (34), L. monocytogenes (35), and S. pneumoniae (36). We also recently demonstrated that the GBS $\beta$-h/c promoted the release of IL- 8 from human lung epithelial cells in vitro (15) and IL-6 in the blood and joint fluid of mice in vivo (6).

The other major GBS virulence factor is the polysaccharide capsule, which acts to resist phagocytosis and thus promotes bacterial survival in the bloodstream. We analyzed HBMEC gene expression during infection with a GBS capsule mutant and found that gene induction was variable compared with the WT infection, but in the case of certain key immune response genes, it was greater than seen with the WT. Our data suggest that the capsule plays a limiting role in the induction of the host inflammatory response. Therefore it may be a mechanism of GBS or other bacterial pathogens to avoid innate immune mechanisms, not only by resisting phagocytosis but also by blunting BBB cytokine secretion. Several animal studies corroborate our findings. For example, purified GBS type III capsule was not required to elicit TNF- $\alpha$ release by human neonatal monocytes (37). Of note, sialic acid, which is present on most human cells, is also found in the GBS capsule as well as capsules of other human meningeal pathogens such as E. coli K1 and $N$. meningitidis groups B and C. It is appealing to speculate that these capsules may represent a form of molecular mimicry whereby the pathogen resembles host cell surfaces to block immune activation.

In summary, we have demonstrated that a specific activation program is induced by a bacterial pathogen in the BBB. Our working model of the acute response of the brain endothelium to GBS includes the upregulation of IL-8, Gro $\alpha$, and Gro $\beta$ for recruitment of neutrophils, GM-CSF for bone marrow stimulation of neutrophils, ICAM-1 for adhesion of neutrophils, and Mcl-1 for prevention of neutrophil apoptosis. This signaling appears to be mediated largely by the bacterial cytolysin $\beta-\mathrm{h} / \mathrm{c}$ and not by the presence of capsular polysaccharide. Our in vivo studies indicate that the $\beta-h / c$ toxin contributes to the development and severity of GBS meningitis. Thus, we speculate that the net effect of the cytotoxic and proinflammatory properties of this toxin works to the detriment of the host. Ongoing studies on the modulation of host gene expression and innate defense mechanisms by meningeal pathogens are critical for understanding early activation of the $\mathrm{BBB}$ and developing preventative therapies for CNS infection.

\section{Acknowledgments}

The authors are grateful to Monique Stins and Kwang Sik Kim for providing HBMEC and to Craig Rubens 
for providing the GBS capsule mutant HY106. The microarray analysis and real-time kinetic RT-PCR were performed at the Center for AIDS Research Genomics Core Facility of the University of California San Diego (Jacques Corbeil, Director; NIH grant AI3614-05) and the Veteran's Medical Research Foundation. Nissi Varki performed histopathologic analysis. This work was supported by a Burroughs Wellcome Fund Career Award (to K.S. Doran), a Howard Hughes Medical Institute Fellowship (to G.Y. Liu), NIH grant HD-37224, the United Cerebral Palsy Research Foundation, and the Edward J. Mallinckrodt, Jr. Foundation.

1. Edwards, M.S., et al. 1985. Long-term sequelae of group B streptococcal meningitis in infants. J. Pediatr. 106:717-722.

2. Ferrieri, P., Burke, B., and Nelson, J. 1980. Production of bacteremia and meningitis in infant rats with group B streptococcal serotypes. Infect. Immun. 27:1023-1032.

3. Nizet, V., et al. 1997. Invasion of brain microvascular endothelial cells by group B streptococci. Infect. Immun. 65:5074-5081.

4. Nizet, V., et al. 1996. Group B streptococcal beta-hemolysin expression is associated with injury of lung epithelial cells. Infect. Immun. 64:3818-3826

5. Gibson, R.L., Nizet, V., and Rubens, C.E. 1999. Group B streptococcal beta-hemolysin promotes injury of lung microvascular endothelial cells. Pediatr. Res. 45:626-634.

6. Puliti, M., et al. 2000. Severity of group B streptococcal arthritis is correlated with beta-hemolysin expression. J. Infect. Dis. 182:824-832.

7. Ring, A., et al. 2002. Group B streptococcal hemolysin induces mortality and liver injury in experimental sepsis. J. Infect. Dis. 185:1745-1753.

8. Leib, S.L., and Tauber, M.G. 1999. Pathogenesis of bacterial meningitis. Infect. Dis. Clin. North Am. 13:527-548.

9. Yim, H.H., Nittayarin, A., and Rubens, C.E. 1997. Analysis of the capsule synthesis locus, a virulence factor in group B streptococci. Adv. Exp. Med. Biol. 418:995-997.

10. Pritzlaff, C.A., et al. 2001. Genetic basis for the beta-haemolytic/cytolytic activity of group B Streptococcus. Mol. Microbiol. 39:236-247.

11. Stins, M.F., Prasadarao, N.V., Zhou, J., Arditi, M., and Kim, K.S. 1997. Bovine brain microvascular endothelial cells transfected with SV40-large $\mathrm{T}$ antigen: development of an immortalized cell line to study pathophysiology of CNS disease. In Vitro Cell. Dev. Biol. Anim. 33:243-247.

12. Huang, S.H., Stins, M.F., and Kim, K.S. 2000. Bacterial penetration across the blood-brain barrier during the development of neonatal meningitis. Microbes Infect. 2:1237-1244.

13. Kim, K.S. 2001. Escherichia coli translocation at the blood-brain barrier. Infect. Immun. 69:5217-5222.

14. Sasik, R., Calvo, E., and Corbeil, J. 2002. Statistical analysis of high-density oligonucleotide arrays: a multiplicative noise model. Bioinformatics. 18:1633-1640.

15. Doran, K.S., Chang, J.C., Benoit, V.M., Eckmann, L., and Nizet, V. 2002. Group B streptococcal $\beta$-hemolysin/cytolysin promotes invasion of human lung epithelial cells and the release of interleukin-8. J. Infect. Dis. 185:196-203.

16. Eckmann, L., et al. 1993. Differential cytokine expression by human intestinal epithelial cell lines: regulated expression of interleukin 8 . Gastroenterology. 105:1689-1697.

17. Todd, M.M., Weeks, J.B., and Warner, D.S. 1992. Cerebral blood flow, blood volume, and brain tissue hematocrit during isovolemic hemodilution with hetastarch in rats. Am. J. Physiol. 263:H75-H82.

18. Kozopas, K.M., Yang, T., Buchan, H.L., Zhou, P., and Craig, R.W. 1993 MCL1, a gene expressed in programmed myeloid cell differentiation, has sequence similarity to BCL2. Proc. Natl. Acad. Sci. U. S. A. 90:3516-3520.

19. Tsao, N., Chang, W.W., Liu, C.C., and Lei, H.Y. 2002. Development of hematogenous pneumococcal meningitis in adult mice: the role of TNFalpha. FEMS Immunol. Med. Microbiol. 32:133-140.

20. Revel, A.T., Talaat, A.M., and Norgard, M.V. 2002. DNA microarray analysis of differential gene expression in Borrelia burgdorferi, the Lyme disease spirochete. Proc. Natl. Acad. Sci. U. S. A. 99:1562-1567.

21. Blader, I.J., Manger, I.D., and Boothroyd, J.C. 2001. Microarray analysis reveals previously unknown changes in Toxoplasma gondii-infected human cells. J. Biol. Chem. 276:24223-24231.

22. Detweiler, C.S., Cunanan, D.B., and Falkow, S. 2001. Host microarray analysis reveals a role for the Salmonella response regulator phoP in human macrophage cell death. Proc. Natl. Acad. Sci. U. S. A. 98:5850-5855.

23. Ichikawa, J.K., et al. 2000. Interaction of Psendomonas aeruginosa with epithelial cells: identification of differentially regulated genes by expression microarray analysis of human cDNAs. Proc. Natl. Acad. Sci. U. S. A. 97:9659-9664.

24. Krishnaswamy, G., Kelley, J., Yerra, L., Smith, J.K., and Chi, D.S. 1999. Human endothelium as a source of multifunctional cytokines: molecular regulation and possible role in human disease. J. Interferon Cytokine Res. 19:91-104.

25. Baggiolini, M., Dewald, B., and Moser, B. 1994. Interleukin-8 and related chemotactic cytokines - CXC and CC chemokines. Adv. Immunol. 55:97-179.

26. Male, D., Rahman, J., Pryce, G., Tamatani, T., and Miyasaka, M. 1994. Lymphocyte migration into the CNS modelled in vitro: roles of LFA-1, ICAM-1 and VLA-4. Immunology. 81:366-372.

27. Hart, M.N., et al. 1992. Brain microvascular smooth muscle and endothelial cells produce granulocyte macrophage colony-stimulating factor and support colony formation of granulocyte-macrophage-like cells. Am. J. Pathol. 141:421-427.

28. Brach, M.A., deVos, S., Gruss, H.J., and Herrmann, F. 1992. Prolongation of survival of human polymorphonuclear neutrophils by granulocytemacrophage colony-stimulating factor is caused by inhibition of programmed cell death. Blood. 80:2920-2924.

29. Coxon, A., Tang, T., and Mayadas, T.N. 1999. Cytokine-activated endothelial cells delay neutrophil apoptosis in vitro and in vivo. A role for granulocyte/macrophage colony-stimulating factor. J. Exp. Med. 190:923-934.

30. Saba, S., Soong, G., Greenberg, S., and Prince, A. 2002. Bacterial stimulation of epithelial G-CSF and GM-CSF expression promotes PMN survival in CF airways. Am. J. Respir. Cell Mol. Biol. 27:561-567.

31. Epling-Burnette, P.K., et al. 2001. Cooperative regulation of Mcl-1 by Janus kinase/stat and phosphatidylinositol 3-kinase contribute to granulocyte-macrophage colony-stimulating factor-delayed apoptosis in human neutrophils. J. Immunol. 166:7486-7495.

32. Bogdan, I., Leib, S.L., Bergeron, M., Chow, L., and Tauber, M.G. 1997. Tumor necrosis factor-alpha contributes to apoptosis in hippocampal neurons during experimental group B streptococcal meningitis. J. Infect. Dis. 176:693-697.

33. Kim, K.S., Wass, C.A., and Cross, A.S. 1997. Blood-brain barrier permeability during the development of experimental bacterial meningitis in the rat. Exp. Neurol. 145:253-257.

34. Thorpe, C.M., Smith, W.E., Hurley, B.P., and Acheson, D.W. 2001. Shiga toxins induce, superinduce, and stabilize a variety of $\mathrm{C}-\mathrm{X}$ - $\mathrm{C}$ chemokine mRNAs in intestinal epithelial cells, resulting in increased chemokine expression. Infect. Immun. 69:6140-6147.

35. Rose, F., et al. 2001. Human endothelial cell activation and mediator release in response to Listeria monocytogenes virulence factors. Infect. Immun. 69:897-905.

36. Rijneveld, A.W., et al. 2002. Roles of interleukin-6 and macrophage inflammatory protein-2 in pneumolysin-induced lung inflammation in mice. J. Infect. Dis. 185:123-126.

37. Vallejo, J.G., Baker, C.J., and Edwards, M.S. 1996. Roles of the bacterial cell wall and capsule in induction of tumor necrosis factor alpha by type III group B streptococci. Infect. Immun. 64:5042-5046. 